\title{
Comparison of High Field Electron Transport in GaAs, InAs and $\mathrm{In}_{0.3} \mathrm{Ga}_{0.7} \mathrm{As}$
}

\author{
B. Bouazza, A. Guen-Bouazza, C. Sayah, N. E. Chabane-Sari \\ Unite de Recherches Matériaux et Energies Renouvelables, Faculté des Sciences de l'Ingénieur, \\ Université Abou-Bekr-Belkaïd de Tlemcen, Tlemcen, Algérie \\ Email: bouaguen@yahoo.fr
}

Received January 8, 2013; revised February 10, 2013; accepted February 25, 2013

Copyright (C) 2013 B. Bouazza et al. This is an open access article distributed under the Creative Commons Attribution License, which permits unrestricted use, distribution, and reproduction in any medium, provided the original work is properly cited.

\begin{abstract}
An ensemble Monte Carlo simulation is used to compare high field electron transport in bulk GaAs, InAs and $\mathrm{In}_{0.3} \mathrm{Ga}_{0.7} \mathrm{As}$. In particular, velocity overshoot and electron transit times are examined. We find the steady state velocity of the electrons is the most important factor determining transit time over distances longer then $0.2 \mu \mathrm{m}$. Over shorter distances velocity overshoot effects in InAs and $\mathrm{In}_{0.3} \mathrm{Ga}_{0.7}$ As at high fields are comparable to those in GaAs. We estimate the minimum transit time across a $1 \mu \mathrm{m}$ InAs sample to be about $4.2 \mathrm{ps}$. Similar calculations for $\operatorname{In}_{0.3} \mathrm{Ga}_{0.7} \mathrm{As}$ yield 6 ps (for GaAs yield $10 \mathrm{ps).} \mathrm{Calculations} \mathrm{are} \mathrm{made} \mathrm{using} \mathrm{a} \mathrm{nonparabolic} \mathrm{effective} \mathrm{mass} \mathrm{energy} \mathrm{band} \mathrm{model,} \mathrm{Monte}$ Carlo simulation that includes all of the major scattering mechanisms. The band parameters used in the simulation are extracted from optimized pseudopotential band calculations to ensure excellent agreement with experimental information and $a b$ initio band models.
\end{abstract}

Keywords: Monte Carlo Method; Semiconductor Devices; Velocity Overshoot; Electron Transport

\section{Introduction}

The Ensemble Monte Carlo technique has been used now for over 30 years as a numerical method to simulate nonequilibrium transport in semiconductor materials and devices, and has been the subject of numerous books and reviews. In application to transport problems, a random walk is generated to simulate the stochastic motion of particles subject to collision processes in some medium. This process of random walk generation may be used to evaluate integral equations and is connected to the general random sampling technique used in the valuation of multi-dimensional integrals. The basic technique is to simulate the free particle motion (referred to as the free flight) terminated by instantaneous random scattering events. The Monte Carlo algorithm consists of generating random free flight times for each particle, choosing the type of scattering occurring at the end of the free flight, changing the final energy and momentum of the particle after scattering, and then repeating the procedure for the next free flight. Sampling the particle motion at various times throughout the simulation allows for the statistical estimation of physically interesting quantities such as the single particle distribution function, the average drift velocity in the presence of an applied electric field, the average energy of the particles, etc. By simulating an ensemble of particles, representative of the physical system of interest, the non-stationary time-dependent evolution of the electron and hole distributions under the influence of a time-dependent driving force may be simulated. The particle-based picture, in which the particle motion is decomposed into free flights terminated by instantaneous collisions, is basically the same picture underlying the derivation of the semi-classical BTE. In fact, it may be shown that the one-particle distribution function obtained from the random walk Monte Carlo technique satisfies the BTE for a homogeneous system in the long-time limit [1].

The purpose of this work is to compare, using Mont Carlo simulation, the potentialities of n-type GaAs, InAs and $\mathrm{In}_{0.3} \mathrm{Ga}_{0.7} \mathrm{As}$. We first analyse, in Section 1 , we explain how to apply our band structure model to Monte Carlo simulation. In Section 2 we present to describe in the detail our calculation of high field transport properties of n-type GaAs, InAs and $\mathrm{In}_{0.3} \mathrm{Ga}_{0.7}$ As by Monte Carlo method. We shall also compare our results with other theoretical and experimental data insofar as it is possible highlight the accuracy of the simulation results. 


\subsection{Free Flight Generation}

In the Monte Carlo method, the dynamics of particle motion is assumed to consist of free flights terminated by instantaneous scattering events, which change the momentum and energy of the particle. To simulate this process, the probability density $p(t)$ is required, in which $p(t) \partial t$ is the joint probability that a particle will arrive at time $t$ without scattering after the previous collision at $t=0$, and then suffer a collision in a time interval $\partial t$ around time $t$. The probability of scattering in the time interval $\partial t$ around $t$ may be written as $\lambda[k(t)] \partial t$, where $\lambda[k(t)]$ is the scattering rate of an electron or hole of wave vector $k$. The scattering rate, $\lambda[k(t)]$, represents the sum of the contributions from each individual scattering mechanism, which are usually calculated using perturbation theory, as described later. The implicit dependence of $\lambda[k(t)]$ on time reflects the change in $k$ due to acceleration by internal and external fields [2-4]. For electrons subject to time independent electric and magnetic fields, the time evolution of $k$ between collisions as

$$
k(t)=k(0)-\frac{e(E+v \times B) \cdot t}{\hbar}
$$

where $E$ the electric field, $v$ is the electron velocity, and $\beta$ is the magnetic flux density. In terms of the scattering rate, $\lambda[k(t)]$, the probability that a particle has not suffered a collision after a time $t$ is given by

$$
\exp \left[-\int_{0}^{t} \lambda\left[k\left(t^{\prime}\right)\right] \cdot \partial t^{\prime}\right] .
$$

Thus, the probability of scattering in the time interval $\partial t$ after a free flight of time $t$ may be written as the joint probability

$$
P_{f}(t) \partial t=\lambda[k(t)] \cdot \exp \left[-\int_{0}^{t} \lambda\left(k_{t^{\prime}}\right) \partial t^{\prime}\right] \cdot \partial t
$$

Random flight times may be generated according to the probability density $p(t)$ above using, for example, the pseudo-random number generator implicit on most modern computers, which generate uniformly distributed random numbers in the range [0,1]. Using a direct method, random flight times sampled from $p(t)$ may be generated according to

$$
r=\int_{0}^{t_{f}} p_{f}\left(t^{\prime}\right) \partial t^{\prime}=\int_{0}^{r_{1}} p\left(r_{1}^{\prime}\right) \partial r_{1}^{\prime}
$$

where $r$ is a uniformly distributed random number and $t_{f}$ is the desired free flight time. Integrating (3) with $p(t)$ given by (2) above yields

$$
r_{1}=1-\exp \left[-\int_{0}^{t_{f}} \lambda\left[k\left(t^{\prime}\right)\right] \cdot \partial t^{\prime}\right]
$$

Since $1-r$ is statistically the same as $r$, (4) may be simplified to

$$
-\ln r_{1}=\int_{0}^{t_{f}} \lambda\left[k\left(t^{\prime}\right)\right] \cdot \partial t^{\prime}
$$

Equation (5) is the fundamental equation used to generate the random free flight time after each scattering event, resulting in a random walk process related to the underlying particle distribution function. If there is no external driving field leading to a change of $k$ between scattering events, the time dependence vanishes, and the integral is trivially evaluated. In the general case where this simplification is not possible, it is expedient to introduce the so called self-scattering method $[1-3,5,6]$, in which we introduce a fictitious scattering mechanism whose scattering rate always adjusts itself in such a way that the total (self-scattering plus real scattering) rate is a constant in time

$$
\Gamma_{0}=\lambda\left(\left[k\left(t^{\prime}\right)\right]\right)+\lambda_{\text {self }}
$$

where $\lambda_{\text {self }}$ is the self-scattering rate. The self-scattering mechanism itself is defined such that the final state before and after scattering is identical. Hence, it has no effect on the free flight trajectory of a particle when selected as the terminating scattering mechanism, yet results in the simplification of (5) such that the free flight is given by

$$
t_{f}=-\frac{1}{\Gamma_{0}} \ln \left(r_{1}\right)
$$

The constant total rate (including self-scattering) $\Gamma_{0}$ is chosen a priori so that it is larger than the maximum scattering encountered during the simulation interval. In the simplest case, a single value is chosen at the beginning of the entire simulation (constant gamma method), checking to ensure that the real rate never exceeds this value during the simulation. Other schemes may be chosen that are more computationally efficient, and which modify the choice of $\Gamma_{0}$ at fixed time increments.

\subsection{Selection of Scattering Rate}

When the electrons are accelerated and the scattering time is chosen, scattering must then occur at the end of the scattering time period. The method used for this is the rejection technique. This technique chooses the scattering using the relative probabilities of the individual events. To start we construct a scattering table and normalize all scattering probabilities to the maximum scattering value, which was found in the above section for the self scattering [4]. Once the entire table is constructed, it can be used throughout the entire simulation without need for recompilation. The selection of the scattering now becomes a two-part step. As the table has already been 
normalized to one, we may use a uniform random number to select the scattering rate. The reason why this works is simple. The choice of scattering is random, but is also governed by the relative strength of certain scattering rates in connection with others that exist in the system. The random numbers take care of choosing the scattering rate, and the relative strength of each scattering rate to the total in the table controls the frequency of certain events over others.

\subsection{Scattering Angle and Final State}

For elastic scattering, the scattering is isotropic. Therefore, all final states in the energy-conserving sphere have the same probability of occupation after scattering. The final angle is independent of the initial state $k$, and the angles of $k^{\prime}$ are proportional to $\sin \theta \cdot \partial \theta \cdot \partial \varphi$. Realizing that the azimuthal angle varies between 0 and $2 \pi$, a direct technique can be employed to obtain $[2,4,7,8]$ :

$$
\cos \theta=1-r_{3} \text { and } \varphi=2 \pi \cdot r_{4}
$$

\subsection{Mean Velocity and Energy Calculation}

When the electric field is applied in the $x$ direction, the average drift velocity and the average electron energy are given for each valley, respectively, by [9],

$$
\left\{\begin{array}{l}
v_{d}=\frac{1}{N} \sum_{i=1}^{N} v_{i}(t) \\
\varepsilon=\frac{1}{N} \sum_{i=1}^{N} \varepsilon_{i}(t)
\end{array}\right.
$$

where

$$
\begin{aligned}
& v_{i}(t)=\frac{\hbar k_{x i}}{m^{*}\left(1+2 \alpha \varepsilon_{i}\right)} \\
& \varepsilon_{i}(t)=\frac{-1+\sqrt{1+4 \alpha \gamma}}{2 \alpha}
\end{aligned}
$$

and

$$
\gamma(k)=\frac{\hbar^{2}}{2 m}\left(k_{x i}^{2}+k_{y i}^{2}+k_{z i}^{2}\right)
$$

where $v_{i}(t)$ and $\varepsilon_{i}(t)$ represent the electron drift velocity and the electron energy at the end of each time step, while $k_{x i}, k_{y i}$ and $k_{z i}$ are the wave vector components in $x, y$ and $z$ direction for each electron, respectively.

\section{Simulation and Results}

In each simulation, twenty thousand electrons are initially distributed in the sample according to an equilibrium Maxwellian distribution at $300 \mathrm{~K}$. A variety of field strengths are simulated to determine the effect on the transient behavior of the electron ensemble. The simula- tion steps the electric field from zero to full intensity at the beginning of the run $(t=0)$, after which the velocity of the electrons is averaged at $10 \mathrm{fs}$ intervals. The average traveled as a function of time is found by integrating the drift velocity. Our Monte Carlo program is based on a three isotropic and non parabolic valley model. The parameters for valleys are estimated from recent band structure calculations. The scattering mechanisms included in the simulations are polar optical phonon, acoustic phonons, piezoelectric, intervalley scattering, ionized impurities and alloy scattering. Values for the various coupling constants which determine many of the scattering rates are the same as those used in Reference [7]. The donor concentration is set to $1.10^{7} / \mathrm{cm}^{3}$.

Figure 1 shows electron velocity versus distance for GaAs, InAs and $\mathrm{In}_{0.3} \mathrm{Ga}_{0.7}$ As. Previous Monte Carlo studies of velocity overshoot in GaAs have performed and are in agreement with the present results. We find the fields which produce the highest steady state velocities (2 $\mathrm{kv} / \mathrm{cm}$ in InAs and $5 \mathrm{kv} / \mathrm{cm}$ in $\operatorname{In}_{0.3} \mathrm{Ga}_{0.7} \mathrm{As}$ ) are similar to the results using the full band Monte Carlo simulation. Furthermore, in all three materials overshoot only occurs at field strengths larger than the peak steady state velocity field and one sees that the higher amplitude of velocity overshoot, the lower its distance (or duration). These observations are tentatively explained in the following manner. First of all, we have checked that, as long as all electrons remain in the $\Gamma$ valley, the velocity increases. Thus, the maximum velocity is reached when the "most rapid" electrons have gained enough energy to transfer to L valley. These electrons are "lucky electrons", which have suffered no, or very few, or very inefficient scattering events. Therefore, the time needed to reach the maximum velocity is mainly determined quasiballistic motion and is sensitive to the scattering rates. On the contrary, the final static velocity is obtained when the whole electron distribution has reached its new equilibrium situation. This process is completed when even "unlucky" widely scattered, electrons have gained enough energy to transfer.

Figure 2 shows the electron transit time as a function of distance traveled. The field strengths transit time occurs when the steady state velocity is the highest. Using the relation $f_{T}=1 /(2 \cdot \pi \cdot \tau)$ where $\tau$ is the transit time at $1 \mu \mathrm{m}$, we estimate the corresponding cutoff frequencies for GaAs to $29 \mathrm{GHz}$. Values as high as $20 \mathrm{GHz}$ chosen minimize the electron transit time at $1 \mu \mathrm{m}$. In GaAs, InAs and $\mathrm{In}_{0.3} \mathrm{Ga}_{0.7} \mathrm{As}$ the minimum have been measured in modern $1 \mu \mathrm{m}$ GaAs modulation doped field effect transistors, not far from the upper limit predicted from the transit time alone.

In Figure 3 we show the transit time as a function of distance in the overshoot regime. In this figure the applied fields were chosen to minimize the transit time 

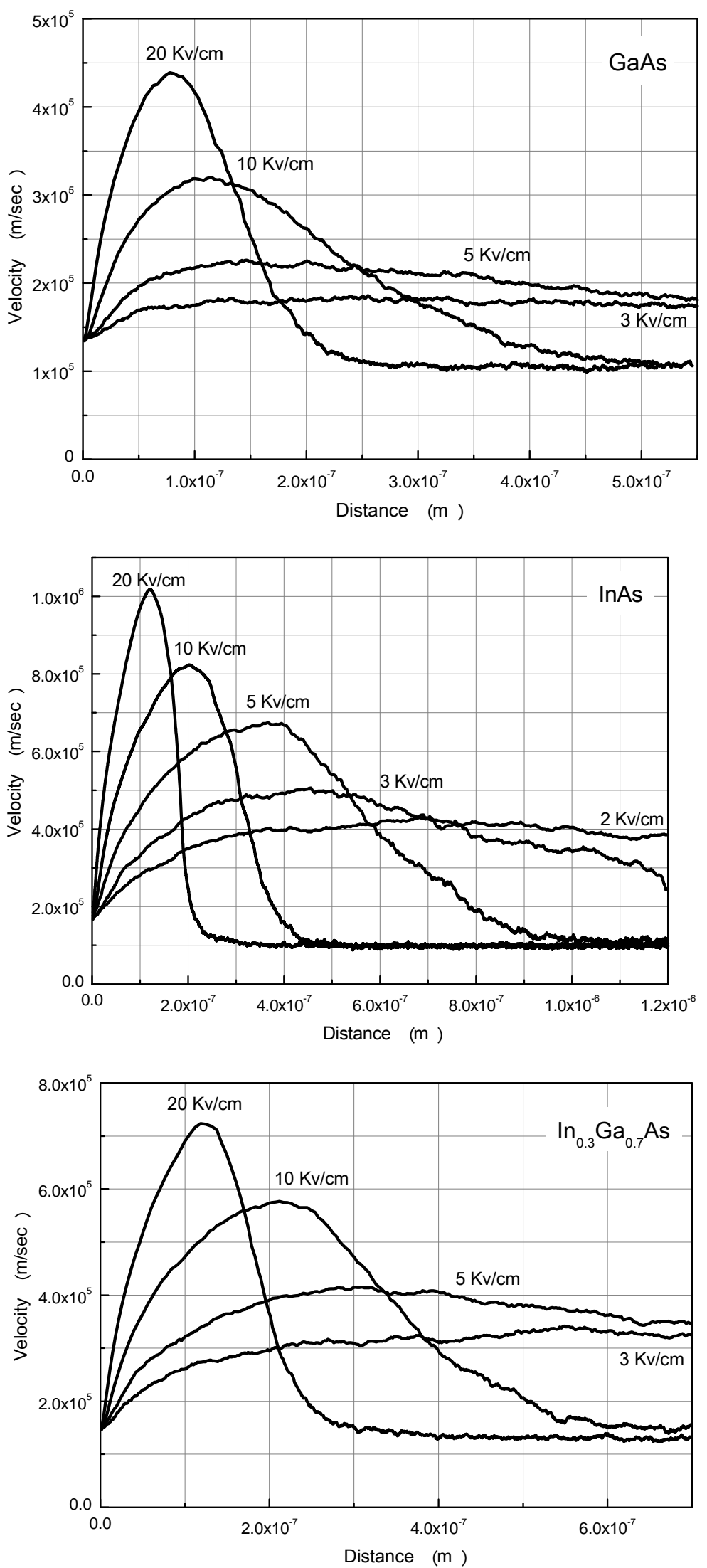

Figure 1. Electron velocity as function of distance in each of the materials simulated. 


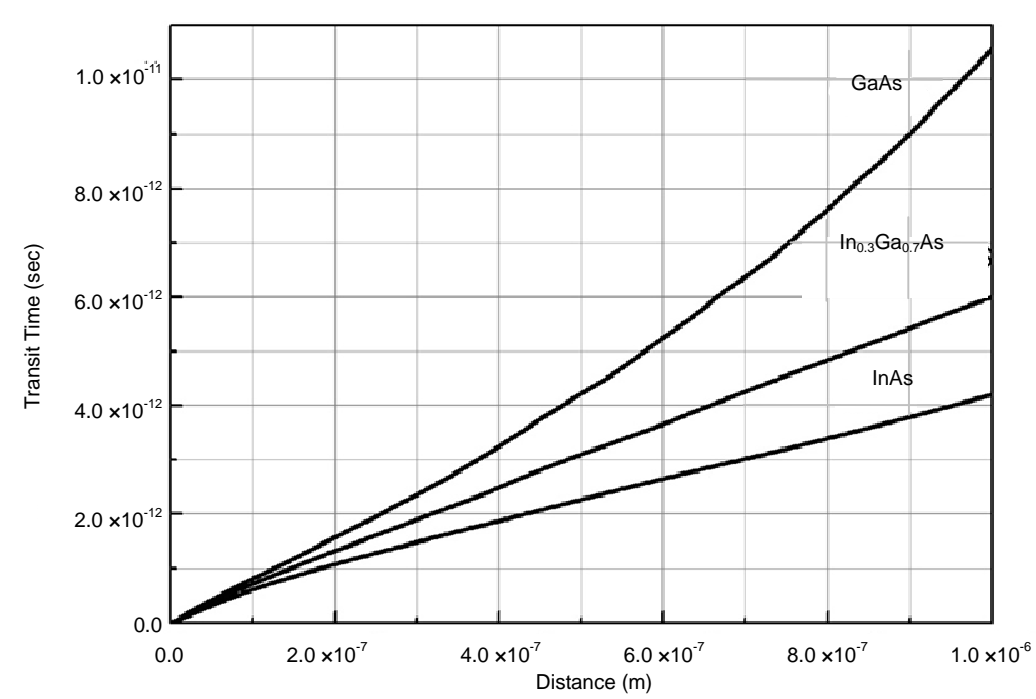

Figure 2. Electron transit time as a function of distance. The field strengths chosen minimize the transit time across $1 \mu \mathrm{m}$. The applied fields are $5 \mathrm{kV} / \mathrm{cm}$ for $\mathrm{GaAs}, 2 \mathrm{kV} / \mathrm{cm}$ for $\mathrm{InAs}$, and $5 \mathrm{kV} / \mathrm{cm}$ for $\mathrm{In}_{0.3} \mathrm{Ga}_{0.7} \mathrm{As}$.

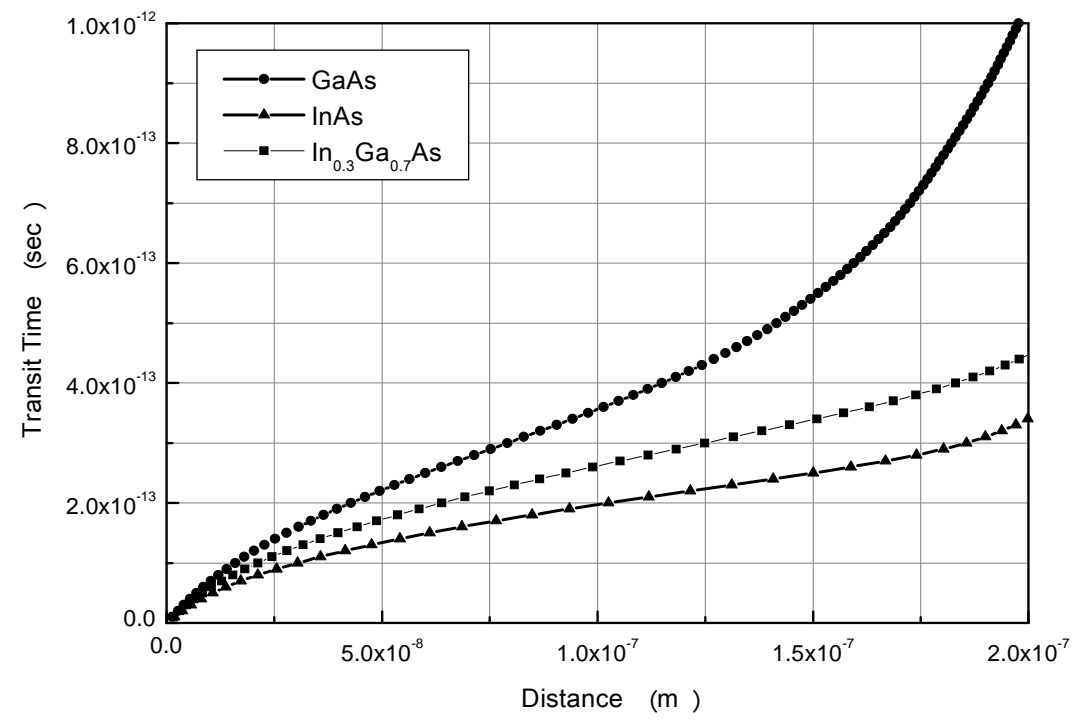

Figure 3. Electron transit time as a function of distance $0.2 \mu \mathrm{m}$. The field strengths chosen minimize the transit time across $0.1 \mu \mathrm{m}$. The applied fields are $20 \mathrm{kV} / \mathrm{cm}$ for GaAs, InAs, and In0.3Ga0.7As.

across a $0.1 \mu \mathrm{m}$ region. In this regime, one normally expects electrons with a lower effective mass to have greter acceleration and therefore have greater velocity and a smaller transit time. We predict however, that although the effective mass is larger InAs

$\left(\mathrm{In}_{0.3} \mathrm{Ga}_{0.7} \mathrm{As}\right)$ its ability to operate at higher voltages allows the transit time to be reduced below that of GaAs. We therefore conclude that for device lengths less than $0.2 \mu \mathrm{m}$ where velocity overshoot is important, the electronic transport properties of GaAs demonstrate no advantage over those of InAs $\left(\operatorname{In}_{0.3} \mathrm{Ga}_{0.7} \mathrm{As}\right)$.

\section{Conclusion}

The authors experience has shown that the effective mass of the gamma valley and the relative energy gap between the valleys has the greatest effect on velocity overshoot and mobility. These parameters have been measured experimentally or have been obtained through band structure calculations. Several of scattering rates depend upon coupling constants that are currently not well known. Therefore, this constant for the acoustic deformation potential was varied by $\pm 20 \%$ and $\pm 40 \%$. The Monte Carlo technique has been used to compare transit times and velocity overshoot effects in GaAs, InAs and $\operatorname{In}_{0.3} \mathrm{Ga}_{0.7} \mathrm{As}$. We find that over distances longer than $0.2 \mu \mathrm{m}$ the transit times in InAs $\left(\operatorname{In}_{0.3} \mathrm{Ga}_{0.7} \mathrm{As}\right)$ are less than those in GaAs due to InAs's ( $\operatorname{In}_{0.3} \mathrm{Ga}_{0.7} \mathrm{As}$ 's) greater peak velocity. Over shorter distances velocity overshoot effects dominate and 
the transit time in InAs $\left(\operatorname{In}_{0.3} \mathrm{Ga}_{0.7} \mathrm{As}\right)$ is comparable or even less than that of GaAs. We conclude that InAs ( $\left.\mathrm{In}_{0.3} \mathrm{Ga}_{0.7} \mathrm{As}\right)$ devices should be capable of equal or higher frequency performance than GaAs when transit time is an important factor.

\section{REFERENCES}

[1] D. Vasileska and S. M. Goodnick, "Computational Electronics," Department of Electrical Engineering, Arizona State University, Tempe, 2006.

[2] L. Shifren, "Ensemble Monte Carlo Study Ultrafast Phenomena Due to Hot Photo-Excited Carriers in bulk GaAs," Thesis for the Degree Master of Science, Arizona State University, Arizona, 1998.

[3] P. Hesto, "Simulation Monte Carlo du Transport Non Stationnaire Dans les Dispositifs Submicroniques, Importance du Phénomène Balistique Dans GaAs à 77 k," Ph.D. Thesis, Université de Paris-Sud, Centre d'Orsay, 1984.

[4] M. Akarsu and Ö. Özbaş, "Osmangazi Monte Carlo Simulation for Electron Dynamics in Semiconductor De- vices," Mathematical and Computational Applications, Vol. 10, No. 1, 2005, pp. 19-26.

[5] C. Moglestue, "Monte Carlo Simulation of Semiconductor Devices," Chapman \& Hall, London, 1993. doi:10.1007/978-94-015-8133-2

[6] C. Jacoboni and P. Lugli, "The Monte Carlo Method for Semiconductor Device Simulation," Springer-Verlag Wien, New York, 1989. doi:10.1007/978-3-7091-6963-6

[7] C. Sayah, "Application de la Méthode de Monte Carlo aux Composés III-V," Thèse de Magister en Electronique, Faculté des Sciences de l'Ingénieur, Université AbouBekr Belkaid, Tlemcen, 2002.

[8] S. Galdin, "Étude du Transistor Dipolaire a Double Hétérojonction $\mathrm{Si} / \mathrm{SiGe} / \mathrm{Si}$ Par Simulation Monte Carlo," Thèse de Docteur en Sciences, Université de Paris-Sud, Centre d'Orsay, 1992.

[9] F. M. Abou El-Ela, "Temperature Dependence of the Transport Properties in ZnS," Egyptian Journal of Solids, Vol. 23, No. 1, 2000, pp. 27-35. 